\title{
Research on the Anti-corona Coating of the Power Transmission Line Conductor
}

\author{
Ziqiang Xu, Ren Li \\ Hebei Provincial Key laboratory of Power Equipment Security Defense, North China Electric Power University, Baoding, China \\ Email: bao1986dan@163.com
}

Received April, 2013

\begin{abstract}
An anti-corona method for the power transmission lines is proposed in this paper. The RTV coating is used as the anti-corona coating, which is spaying onto the surface of the wearing conductor. The corona characteristic of the conductor test was done, and the corona onset voltage increase after the spraying of the anti-corona layer, the corona loss in the same voltage decrease, which could prove the excellent effect of improving the corona characteristic of the conductor. This anti-corona method will have great prospect, based on the background of the construction of UHV power transmission lines.
\end{abstract}

Keywords: Corona Loss; EHV/UHV Power Transmission; RTV Coating; Anti-corona

\section{Introduction}

Corona discharge is a discharge phenomenon that the surface electric field strength exceeds the breakdown electric field strength. The breakdown electric field strength is generally $20-30 \mathrm{kV} / \mathrm{cm}$, when the electric field strength exceeds this value, the discharge sound could be heard, and the smell of ozone, and the bluish velvet light could be seen in the surrounding of the conductor. The ions generated by corona discharge move fro and to the conductor by the alternating electric field, and light and radio interference will be generated in the meantime. The above effect will cause a consumption of energy, which is called corona loss. The calculation of corona loss is the primary work in the optimum transmission line design. Especially the UHV long distance power transmission in the high altitude areas, the calculation of the corona loss plays a decisive role [1-3].

Nowadays, the research on UHV and EHV power transmission is in the studying the characteristic step. Measures like enlarging the diameter of the conductor, enlarging the bundle space or raise the height of the tower can reduce the corona loss of power transmission line, but on the other hand, the measures themselves will cause a lot of money. And for the conductors with defects, it is really unrealistic to replace the original conductors in large scale. It is urgently need to find a new way to reduce the corona loss of the UHV/EHV power transmission lines. The application object of the anti-corona coating is the windings of the power generator, which is different from the high voltage level power transmission line [4-5].

\section{Selection of the Coating}

The research on the anti-corona coating is relatively little, the research mainly aims at the anti-corona of the windings of the power generator. After overall consider of the performance of the coating and the cost, the anti-corona coating should have the following characteristics: low cost, the easy availability and no harm to the environment.

RTV coating is a widely used coating for its good heat and cold resistant and electric insulation property. The good hydrophobicity and migration of hydrophobicity make it have the performance of pollution flashover characteristics, which is the best plan in solving the problem of pollution flashover of the outer insulation of the power equipment.

How to put the coating on the surface of the conductor is another problem, because the shape of the coating will greatly influence the effect of anti-corona. The methods in GBT4585.2-91 artificial pollution test of the high voltage insulators in AC systems: quantitative brush method, pouring method, spray dyeing method, etc. the quantitative brush method and pouring method could not make the surface of the conductor uniform, so in the end spray dyeing method is taken. The conductor with and with no coating are shown in Figure 1 and Figure 2.

The surface electric field strength is calculated with the finite element method with the software ANSYS, the electric field strength decrease with the increase of the thickness of the layer, the relative dielectric constant is 3 , and the electric field strength condition is much improved after spraying the anti-corona coating. The di- 
ameter of the conductor is $22.28 \mathrm{~mm}$. The calculation results are shown in Figure 3 and Figure 4.

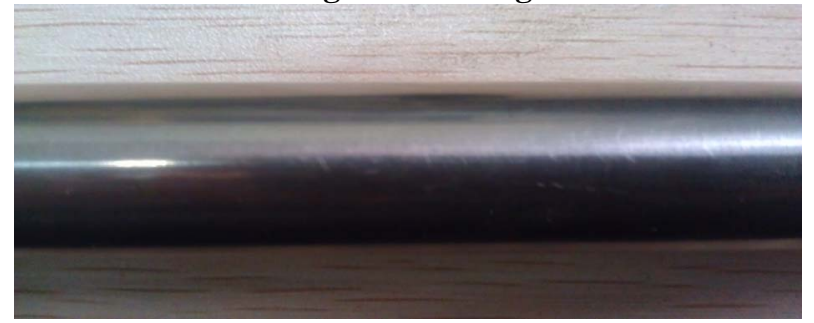

Figure 1. Conductor with no coating

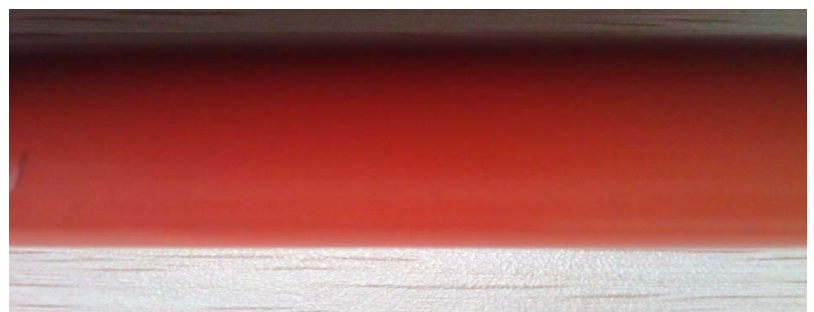

Figure 2. Conductor with coating

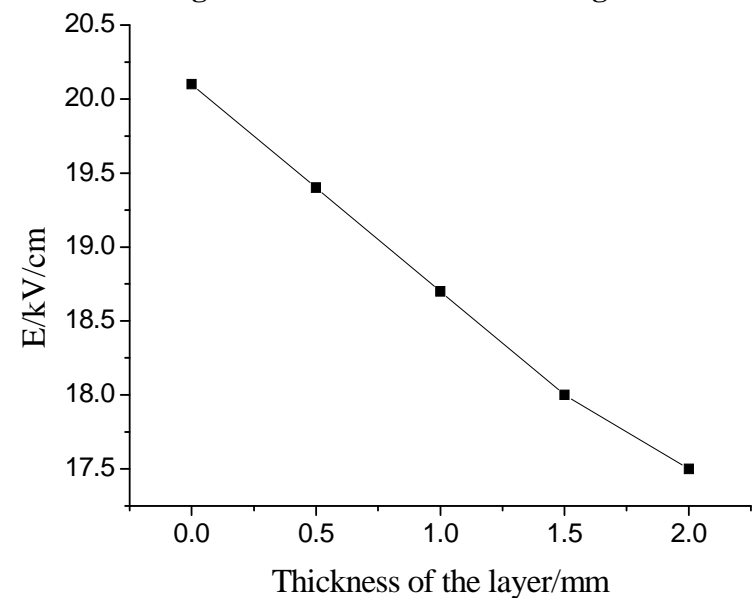

Figure 3. Electric field calculation result.

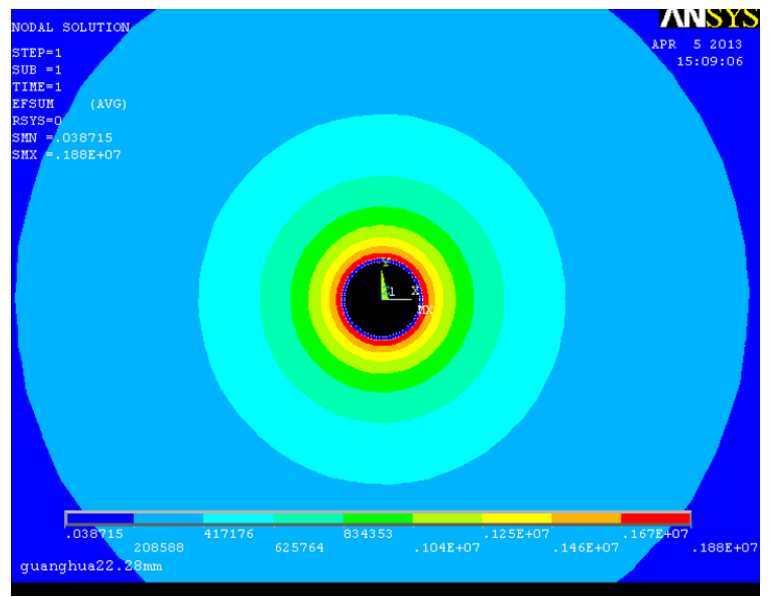

Figure 4. Electric field distribution of the conductor with $1 \mathrm{~mm}$ coating.

\section{Corona Characteristic Test}

\subsection{Test Arrangement}

Corona loss experiments based on the small corona cage is done in North China Electric Power University, key laboratory Hebei Provincial key laboratory of power transmission equipment security defense, the cross-sectional structure size of the corona cage is $1.8 \times 1.8 \mathrm{~m}$, and the length is $6 \mathrm{~m}$. With a rated voltage of test transformer is $250 \mathrm{kV}$, which can satisfy the requirement of corona experiment. The corona loss measurement system through hybrid light-powered electronic current sensors to achieve safe and reliable measurement of the corona current in corona cage; outdoor high-precision capacitive voltage divider can achieve accurate and reliable measurement of the voltage; using the coaxial cable to transmit voltage signals and the optical fiber is used to transmit current signals, combined with modern digital signal processing technology and virtual instrument technology, voltage and current signals are accurately measured. Based on the software Labview, the voltage and current signals are collected and corona loss is calculated. The sketch of the measurement system is shown in Figure 5.

Instantaneous power method is used to calculate the corona loss, curren $i(t)=I_{m} \sin \left(\omega t+\phi_{i}\right)$, voltage $u(t)=U_{m} \sin \left(\omega t+\phi_{i}\right)$, I is the current effective value, $\mathrm{U}$ is the voltage effective value, the power factor angle.

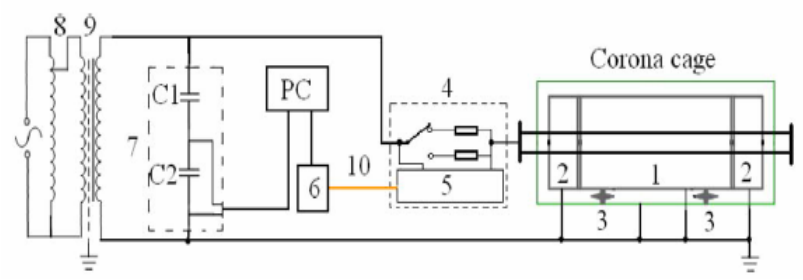

1-measuring segment; 2-shielding segment; 3-support insulation; 4-resistance switch box; 5-OPCT upper module; 6-OPCT local module; 7-capacitive voltage divider; 8-voltage regulator; 9-test transformer; 10- optical fiber

Figure 5. Sketch of the electrical-optical measurement system structure.

$$
P=I U \cos \varphi=\frac{1}{n T} \int_{0}^{n T} i(t) u(t) d_{t}
$$

After the discretization,

$$
P=\frac{1}{n f_{s} T} \sum_{j=0}^{n f_{s} T-1} i(j) u(j)
$$

where, $f_{s}$ is sampling frequency, $\mathrm{N}$ is computing cycles

\subsection{Test Procedure}

The first experiment is done on corona loss without coating material wire. In order to damage in the surface, grinding the wire surface with coarse sandpaper, then 
spray dyeing coating on the wire and the thickness of the layer is measured by vernier caliper, coating thickness is about $1 \mathrm{~mm}$, and the experiment is done on the conductor with coating and without coating.

The measurement results are shown in Figure 6. As can be seen, conductor corona loss decreases with the increase of coating thickness. From the point of the corona loss changes, the corona inception voltage value can be seen roughly, in the corona inception voltage can be found to increase with the wire thickness. The anti-corona coating effect is obvious; the thickness of the coating should depend on the practical situation of the conductor.

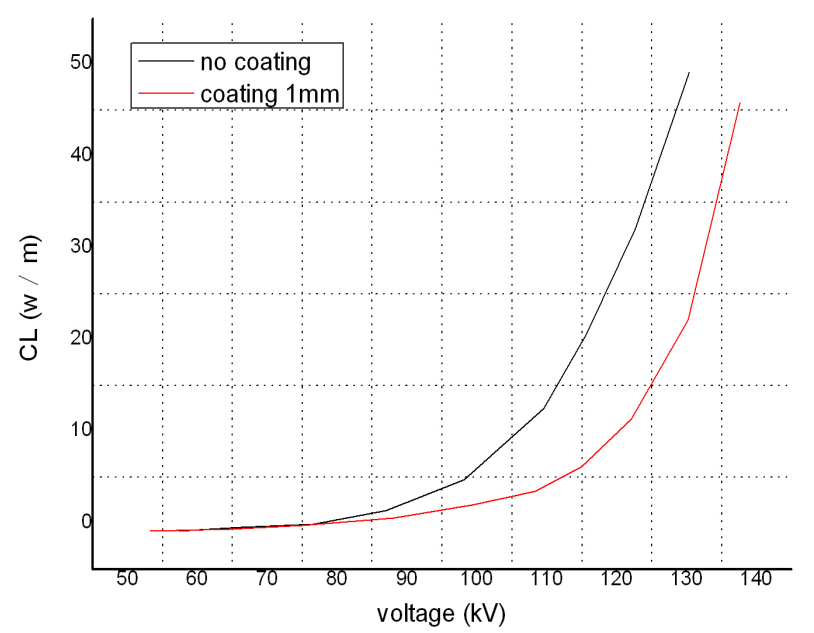

Figure 6. Measurement results.

\section{Conclusions}

An anti-corona method is proposed in this paper by spaying the RTV coating on the wear conductor.

The electric field strength with the coating is less than that with no coating, and the thicker the coating is, the less the electric field strength becomes.

The corona loss of the conductor with RTV coating is less than that with no coating, which means the method proposed in this paper is effective.

\section{REFERENCES}

[1] Z. Y. Liu: “Ultra-high Voltage Grid”, Beijing: China Economic Publishing House, 2005, pp. 253-256.

[2] J. J. Clade and C. H. Gary: "Predetermination of Corona Losses under Rain: Experimental Interpreting and Checking of a Method to Calculate Corona Losses," IEEE Transactions on PAS, Vol. 89, No. 5, 1970, pp. 853-860.

[3] P. S. Maruvada: "Corona performance of high-voltage transmission lines”, London, UK: Research Studies Press Ltd, 2000.

[4] Y. P. Liu, S. H. You, Q. F. Wan, et al., "Design and Realization of AC UHV Corona Loss Monitoring System," High Voltage Engineering, Vol. 34, No. 9, 2008, pp. 1797-1801.

[5] F. C. Lu, S. H. You, Y. P. Liu, Q. F. Wan and Z. B. Zhao, "AC Conductors' Corona-loss Calculation and Analysis," IEEE Transactions on Power Delivery, Vol. 27, No. 2, 2012, pp. 877-885.doi:10.1109/TPWRD.2012.2183681 